\title{
SEGURANÇA ALIMENTAR E NUTRICIONAL EM TEMPOS DE COVID-19: IMPACTOS NA ÁFRICA, AMÉRICA LATINA E PORTUGAL
}

\author{
Maitu Abibo Buanango ${ }^{1}$ \\ Lilian Fernanda Galesi-Pacheco ${ }^{2}$ \\ Yudi Paulina Garcia Ramirez ${ }^{3}$ \\ Cristina Amaro da Costa ${ }^{4}$ \\ Jaqueline Sgarbi Santos 5 \\ Ana Pinto de Loura ${ }^{6}$ \\ Carla Maria Vieira ${ }^{7}$
}

\begin{abstract}
Resumo
Crises econômicas e sanitárias têm grandes repercussões no acesso aos alimentos e saúde da população. Neste contexto, políticas de austeridade podem apresentar efeitos devastadores em mecanismos de garantia de direitos sociais. O objetivo deste ensaio é refletir sobre o impacto de diversos tipos de crises no Direito Humano à Alimentação e Nutrição Adequada (DHANA) e na Segurança Alimentar e Nutricional (SAN) de populações em vulnerabilidade, bem como a identificação das políticas públicas, programas e ações desenvolvidas tomando como referência o alívio dos danos causados pela pandemia da COVID-19. A partir de coleta da informação realizada de forma não sistemática em sites governamentais e bases de dados científicas, documentos foram lidos na íntegra, categorizados e analisados criticamente. Três contextos distintos, África, América Latina e Portugal, são apresentados pelos autores que atuam nessas realidades, com a intencionalidade de contribuir para mitigar os problemas alimentares que se agravam e são derivados da situação pandêmica.
\end{abstract}

Palavras-Chave: Consumo; COVID-19; Direito à Alimentação; Segurança Alimentar e Nutricional; Pandemia.

\section{Introdução}

\footnotetext{
${ }^{1}$ Doutorando na Faculdade de Ciências Farmacêuticas, Universidade Estadual Paulista "Júlio de Mesquita Filho" - UNESP, Campus de Araraquara, São Paulo, Brasil; e Universidade Zambeze - UniZambeze, Beira, Moçambique

${ }^{2}$ Pós-doutoranda no Departamento de Educação do Instituto de Biociências, Letras e Ciências Exatas (IBILCE) Campus de São José do Rio Preto-SP da Universidade Estadual Paulista "Júlio de Mesquita Filho" - UNESP / Membro do Centro de Ciência, Tecnologia e Inovação para Soberania e Segurança Alimentar e Nutricional (INTERSSAN).

${ }^{33}$ Professora da Faculdade de Ciências da Saúde, Corporación Universitaria Remington, Medellin, Colômbia e Faculdade de Ciências dos Alimentos e Nutrição, Universidad CES, Medellin, Colômbia.

${ }^{4}$ Professora na Escola Superior Agrária do Instituto Politécnico de Viseu (PV). CERNAS - Centro de Recursos Naturais, Ambiente e Sociedade, Portugal.

5 Professora do Instituto de Desenvolvimento Rural (IDR) da Universidade da Integração Internacional da Lusofonia Afro Brasileira, Redenção, Ceará.

${ }^{6}$ Professora Auxiliar da Universidade Aberta/GREENUPorto, UP, Portugal. Centro de Investigação em Produção Agroalimentar Sustentável.

${ }^{7}$ Pesquisadora do Centro de Ciência, Tecnologia e Inovação para Soberania e Segurança Alimentar e Nutricional (INTERSSAN); Docente/facilitadora do Instituto de Ensino e Pesquisa Hospital Sírio-Libanês - PROADI-SUIS.
} 
De acordo com balanço da Organização das Nações Unidas (ONU) sobre segurança alimentar no ano de 2019, o número absoluto de pessoas subalimentadas continua crescendo, mesmo que com lentidão. Hoje, mais de 820 milhões de pessoas continuam padecendo de fome no mundo todo e mais de 2000 milhões de pessoas estão em insegurança alimentar moderada ou grave, o que destaca o imenso caminho que permita alcançar o objetivo de fome zero para 2030. A Fome está aumentando em quase todas as sub-regiões de África e, em menor medida, na América Latina e Ásia Ocidental (FAO, 2019). A falta de acesso regular aos alimentos nutritivos e suficientes colocam a população em um risco maior de má-nutrição e de adoecimento (FAO, 2020a).

Crises econômicas, assim como as sanitárias, têm grandes repercussões no acesso aos alimentos tanto quanto na saúde pública da população, principalmente porque as políticas de austeridade podem apresentar efeitos devastadores nos mecanismos estabelecidos de garantia de direitos sociais (GUIMARÃES, 2018).

A austeridade é uma estratégia recente, neoliberalista, que impõe sacrifício pelos cortes de despesas e redução das estruturas estatais (SANTOS; VIEIRA, 2018). Esses cortes costumam ser mais impactantes nas populações mais vulneráveis e que necessitam de proteção social, em grande medida provida pelo Estado.

A preocupação em relação à escassez de alimentos no mercado mundial, dada por restrições à exportação devido a incertezas sobre a disponibilidade de alimentos tem sido reconhecida pela Organização das Nações Unidas para a Alimentação e Agricultura (FAO), no atual cenário da pandemia por COVID-19. Alterações na oferta e demanda e aumento de preços são considerados nos estudos sobre o tema, assim como a necessidade de proteção dos produtores e trabalhadores do setor alimentício no contágio, para manter a cadeia produtiva e o fluxo do comércio de alimentos, o quanto possível (FAO, 2020f). Contudo, os esforços dos países afetados têm sido voltados para evitar a propagação do vírus, possibilitar atendimento em saúde e criar caminhos para apoiar a economia. As medidas de isolamento domiciliar, de fechamento do comércio, de serviços não essenciais, bem como necessidade de realocação de investimentos para o sistema de saúde, já têm provocado significativas consequências no sistema econômico, em muitos casos já fragilizados.

A pandemia da COVID-19 revela profundas lacunas na SAN, exacerbadas à medida que o isolamento social necessário se expande. Com a piora do quadro nutricional dos grupos mais vulneráveis e desprovidos de uma política de proteção social e de distribuição de riquezas, 
são primordiais estudos que possam contribuir com os governos, na implementação de planos de desenvolvimento territorial com metas para mitigar a desnutrição e o aumento da pobreza.

Esta abordagem é tanto mais urgente, considerando que o Pacto Internacional dos Direitos Econômicos, Sociais e Culturais das Nações Unidas (ONU, 1966), conta com 174 países signatários, e esse documento complementado pelo Comentário n. 12 e, em muitos casos, explicitado nas Constituições de muitos países, torna a garantia do Direito Humano à Alimentação Adequada (DHAA) dever do Estado. Sendo assim, o Estado tem que garantir que, mesmo em situações de crise, as políticas envolvendo alimentação e nutrição devem fazer parte dos sistemas de proteção social (BURITY et al, 2010).

Três contextos com suas especificidades e também convergências são apresentados, nomeadamente África, América Latina e Portugal, origens dos autores, e onde estes atuam e têm dado contributo a vários níveis sobre o assunto. Dessa forma, tendo em conta os conhecimentos existentes, fruto de suas experiências e vivências no cotidiano, sustentados pelo manancial teórico, importantes ativos para a compreensão de qualquer fenómeno complexo, surgiu a preocupação para a reflexão do impacto de diversos tipos de crises no DHANA e na SAN de populações em vulnerabilidade, bem como a identificação das políticas públicas, programas e ações desenvolvidas tomando como referência o alívio dos danos causados pela pandemia da COVID-19.

\section{Metodologia}

O presente estudo foi elaborado como um ensaio desenvolvido a partir de uma análise da literatura científica e reflexão crítica dos autores. A proposta deste ensaio está voltada para o levantando questões e busca colaborar com a obtenção e atualização do debate em torno do tema em questão.

O processo de elaboração do texto foi realizado de forma não sistemática no período de abril a junho de 2020. Os autores se dedicaram a fazer atualização da literatura em sites governamentais e bases de dados científicas como Scielo, Scopus, Lilacs e Pubmed, usando descritores "COVID-19”, "SARS-CoV-2", "Segurança Alimentar e Nutricional”, "Direito Humano à Alimentação e Nutrição Adequada", “América Latina”, "Portugal”, “Àfrica”. Os mesmos descritores traduzidos para as línguas inglesa e espanhola. As informações foram complementadas por artigos do acervo pessoal dos autores e conhecimentos tácitos, tendo em vista seus percursos como pesquisadores e suas experiências práticas no campo de atuação que envolve o tema da Segurança Alimentar e Nutricional. 


\section{Garantia do DHAA em países da África frente à COVID-19}

A pandemia da COVID-19 é ainda mais devastadora em países que já enfrentam conflitos, desastres naturais ou mudanças climáticas (ONU, 2020). Um dos exemplos é o de Moçambique, que neste momento encontra-se mergulhado em conflitos armados e violentos nas zonas Centro e Norte do País, resultando em deslocamento de famílias de seu local de origem, deixando para trás seus cultivos e víveres. Somando-se a isso, em 2019, à semelhança de Malawi e Zimbabué, o país registrou a ocorrência de Ciclones Idai e logo a seguir o Kenneth. Essas mudanças climáticas comprometem a agricultura e, com isso, afetam a SAN de muitas famílias (MASIPA, 2017), seja na disponibilidade de alimentos, na garantia do acesso a todos, em diferentes aspectos do consumo e boa nutrição e estabilidade em todas essas dimensões (TONG et al., 2016). É nos sistemas agrícolas de subsistência que esses impactos se mostram mais comprometedores (MENHAS; UMER; SHABBIR, 2015).

$\mathrm{Na}$ África, com a restrição à mobilidade de pessoas em virtude da pandemia de COVID19, teme-se um grande prejuízo na agricultura, que depende intensamente de trabalho humano, além do risco no suprimento de insumos (FAO, 2020d). Neste cenário, as plantações ficarão expostas às pragas e os gafanhotos representam a principal ameaça. Especificamente, são mais comprometidos os produtos perecíveis, vegetais, fruta, e horticulturas, além de carnes e lacticínios, prejudicando sobremaneira a SAN da população africana.

No continente africano destacam-se dois países, por terem sido os primeiros a empreender ações concretas para evitar o fraco acesso aos alimentos em virtude das medidas de restrição e de medidas de isolamento social durante a pandemia, nomeadamente África do Sul e Ruanda. Conforme refere Alencastro (2020), a África do Sul disponibilizou um fundo de 2,2 bilhões de dólares Norte Americanos, o equivalente a 11,24 bilhões de Reais, para ajudar pessoas incapazes de trabalhar e assim tentar evitar a perda de empregos.

Ruanda, por sua vez, de acordo com a African News Agency (2020) formulou um plano de proteção social para a entrega de alimentos gratuitos a pelo menos 20.000 famílias na capital Kigali, com objetivo de limitar a propagação de COVID-19.

\section{Dilemas de países da América Latina frente à COVID-19}

A restrição da circulação das pessoas nas ruas e ambientes coletivos influencia diretamente a renda de trabalhadores autônomos e informais sem contar os que estão em busca de um emprego (ASSOCIAÇÃO BRASILEIRA DE NUTRIÇÃO, 2020). A perspectiva é de 
que mesmo os assalariados tenham seus rendimentos afetados pelo risco de demissão ou redução da jornada de trabalho (OLIVEIRA; ABRANCHES; LANA, 2020). Essa situação também pode ser identificada em países onde o abastecimento alimentar, do mesmo modo que a obtenção de renda, ocorre via mercados e feiras populares, impactadas em tempos de pandemia.

A renda não é o único fator comprometido em classes desfavorecidas. Pode-se destacar também a exposição à contaminação pelo vírus causador da pandemia dada pelo uso de transporte público, aglomerações nas moradias, condições inadequadas de higiene dificultando à adesão às medidas de combate, menor acesso ao saneamento básico e aos serviços de saúde (BURITY, 2020; PIRES, 2020; MACEDO; ORNELLAS; BOMFIM, 2020).

Adiciona-se o risco ao contágio e piora do quadro da COVID-19 entre pessoas em situação de rua que comumente apresentam doenças crônicas sem tratamento, acompanhadas do uso de álcool, drogas e baixíssimo acesso à alimentação de qualidade (PIRES, 2020). Segundo a FIAN - Brasil, os grupos que já são historicamente vítimas de violações de direitos serão os mais afetados pela pandemia (BURITY, 2020). Este cenário é agravado quando se vislumbra o aumento do número de pessoas nessa situação (OLIVEIRA; BRANCHES; LANA, 2020).

Outra parcela da população que está em desvantagem na atual pandemia é a representada pelas mulheres, principalmente as afro-descendentes e com baixa renda, de regiões brasileiras favelizadas (ASBRAN, 2020). A sobrecarga de trabalho doméstico, o cuidado familiar, a exclusão do mercado de trabalho e a violência doméstica, são os principais fatores responsáveis pela vivência conturbada das mulheres nesse período. Há também, o acúmulo de funções pela inserção no mercado de trabalho, como as profissionais de saúde, que representam cerca de $70 \%$ das equipes e estão mais expostas ao contágio da doença (PIRES, 2020).

O Programa Mundial de Alimentos informou que 14 milhões de pessoas podem estar vulneráveis à fome, causada pela crise social e econômica devido a COVID-19 na América Latina (FAO, 2020b).

Diante da crise de saúde gerada pelo COVID-19 e de uma possível diminuição no consumo e impacto na qualidade dos alimentos, as populações com o mais alto grau de desigualdade e com altos níveis de pobreza serão as mais afetadas. Em um relatório da FAO, Comunidade de Estados Latinoamericanos e Caribenhos, FAO (2020e), foi demonstrado que as variações no poder de compra das famílias geram mudanças na demanda por alimentos e no 
comportamento e preferência do consumidor. A taxa do desemprego na Colômbia no final do mês de março de 2020 foi registrado em 12,6\%, representando um aumento de 1,8\% quando comparado ao mesmo período do ano passado. E, em Abril de 2020, aquela taxa subiu para $19,8 \%$, demonstrando aumento de 9,5\% em relação ao igual período do ano anterior (DANE, 2020).

$\mathrm{Na}$ América Latina, diversas instituições acadêmicas, organizações governamentais e da sociedade civil têm se organizado para propor estratégias de enfrentamento da crise econômica gerada pelo novo coronavírus em todos os níveis do sistema alimentar. Na busca, encontramos algumas propostas e estratégias apresentadas brevemente aqui.

O Escritório Regional da FAO para a América Latina e o Caribe, preocupado com a garantia da SAN de crianças com a suspensão do oferecimento da alimentação nas escolas, propôs oito medidas possíveis de serem adotadas pelos países para garantir a alimentação infantil. As propostas envolvem principalmente a garantia de acesso de alimentos às famílias, como estratégias de entrega e de aumento da renda (FAO, 2020g).

Recomenda-se para que haja fornecimento contínuo de refeições às crianças de famílias de baixa renda durante esse período (ONU, 2020), sobretudo para países como que adotaram em suas Constituições a obrigatoriedade do Estado garantir o Direito Humano à Alimentação. No Brasil, o programa de alimentação escolar, existe desde de 1955 (Decreto $\mathrm{n}^{\circ}$ 37.106), contudo foi sua reformulação, ocorrida em 2009 que o tornou uma política estratégica para a SAN no país, com destaque para a inclusão da agricultura familiar como fornecedora de alimentos para o Programa. O isolamento social tem provocado descontinuidade deste benefício e respostas diversas por parte dos municípios. Alguns estão oferecendo um bônus às famílias, outros tem conseguido oferecer alimentos em marmitas que são retiradas nas escolas, mas há municípios que não tomaram qualquer providência nesse sentido. Desta maneira, a interrupção das atividades presenciais escolares fragiliza a SAN de crianças e adolescentes de baixa renda (FAO, 2020b).

Entre os hispânico/latinos, existem comunidades que podem estar mais expostas à COVID-19, em decorrência das condições pré-existentes. A título de exemplo, destacam-se os migrantes e imigrantes que compõem o grupo de maior vulnerabilidade. Ao longo do tempo, observa-se que estes últimos apresentam dificuldades comunicacionais, linguísticas e de adaptação no país de acolhimento. O confronto com o preconceito leva à aceitação de postos de trabalhos pesados e mal remunerados e pouco reconhecidos socialmente. Situação agravada 
pelas fracas redes sociais e dificuldade de informação e acesso aos serviços, designadamente de saúde. Exposição ao isolamento social, aos processos de aculturação e sofrimento psicológico e doença decorrente deste processo, habitação precária e insalubre em regiões desfavorecidas e sujeitas às catástrofes naturais e violência, deficiência alimentar, uso de drogas e álcool, incidência de certas condições de trabalho, entre outros, são fatores que tornam essa população mais vulnerável que as populações locais (CHAVEZ; TELLEEN; KIM, 2007; POTOCHNICK et al., 2019).

No elenco de ações de enfrentamento do atual contexto, podemos citar a carta intitulada "Garantir o direto à alimentação e combater a fome em tempos de coronavírus: a vida e a dignidade humana em primeiro lugar!" que foi subscrita por fóruns, redes, articulações, movimentos e organizações da sociedade civil brasileira que atuam na defesa do DHANA, DHAA e da Soberania e SAN. O documento apresenta onze propostas de combate à fome destinadas às instâncias municipais, estaduais e federal para serem desenvolvidas em parceria com conselhos de participação social adaptadas à realidade de cada local (ASBRAN, 2020).

O Instituto Brasileiro de Defesa do Consumidor (IDEC) criou uma plataforma chamada "Comida de Verdade" para ajudar os consumidores a encontrarem iniciativas que comercializam alimentos saudáveis e sustentáveis e, além disso, oferecer apoio a pequenos produtores escoarem suas produções, durante a pandemia (INSTITUTO BRASILEIRO DE DEFESA DO CONSUMIDOR, 2020).

Outras publicações trazem preocupações com o abastecimento, desperdício e aumento de preços dos alimentos. A preocupação é especialmente voltada à disponibilidade e acesso de alimentos pelas populações em vulnerabilidade social, bem como o agravamento da situação econômica de pequenos produtores. Colocam o incentivo à agricultura familiar, circuito curto de comercialização e ao comércio local com apoio das redes alimentares alternativas, como estratégia para não prejudicar a disponibilidade de alimentos e como forma de oferecer acesso a alimentos frescos e saudáveis (FIOCRUZ 2020; MELLO-THÉRY; THÉRY, 2020; OLIVEIRA; ABRANCHES; LANA, 2020). Conforme dados pesquisados por Lucena, Holanda e Bomfim (2020), investimentos na agricultura familiar são de duas a três vezes mais efetivos para reduzir a pobreza e a insegurança alimentar e ações de Estado para mitigar os impactos nesse setor são extremamente necessárias.

No mesmo sentido, Domingues (2020) trouxe à tona, a preocupação com problemas de abastecimento e de SAN. O autor listou quatorze propostas para reduzir a insegurança alimentar 
como a distribuição de cestas básicas em locais estratégicos, redução do preço do gás, oferecimento de linhas de créditos ao pequeno agricultor para cadeias de produção com incentivo ao comércio local, criação de hortas comunitárias, elaboração de mapas municipais para políticas locais de abastecimento, manutenção das feiras com cuidados que minimizem risco de contágio, organização de redes alternativas e suspensão da privatização da Companhia de Entrepostos e Armazéns Gerais de São Paulo (CEAGESP).

Neste contexto, cabe lembrar as possibilidades de contribuição da agricultura familiar e camponesa no alcance da SAN, em especial em tempos de pandemia. As características essenciais desse tipo de agricultura são altamente debatidas pela literatura mundial e dispensam aqui conceituações, contudo vale lembrar atributos que a colocam num papel estratégico para contribuir em diversas frentes concernentes aos sistemas alimentares, principalmente por sua diversidade, resiliência e capacidade adaptativa (BAIARDI; ALENCAR, 2014).

A agricultura familiar desempenha papel estratégico na promoção da SAN, em tempos de pandemia, com destaque para a utilização de sistemas de produção mais eficientes no uso de recursos naturais, cada vez mais escassos, em grande medida impactados pelas mudanças climáticas. Evidencia-se a capacidade de participar de sistemas de abastecimento de alimentos saudáveis, tendo como base a produção agroecológica e caracterizados pela relação direta entre produtores e consumidores, a exemplo dos mercados populares e feiras-livres.

No nordeste brasileiro, o abastecimento realizado por meio de feiras-livres é algo histórico, enraizado na cultura e tradição local, onde consumidores e vendedores interagem para além da compra e venda de produtos, construindo-se espaços de convivência em que as relações sociais são fortalecidas (FORMAN, 1979). Em tempos de pandemia, os mercados e feiras-livres estão impactados pela necessidade de isolamento que os impede de se manter. Algumas iniciativas nessa região, nomeadamente nos estados da Bahia e Ceará, o poder público disponibilizou plataformas tecnológicas para comercialização de produtos, por meio de sites governamentais com espaço para que produtores possam comercializar seus produtos, facilitando a aproximação dos produtores e consumidores. Também são identificadas experiências de famílias de produtores, tradicionalmente feirantes, que estão conseguindo superar os entraves estruturais e se reinventam, construindo estratégias de entrega de produtos à domicílio, pois já contavam com pontes alicerçadas em relações de confiança e reciprocidade construídas na feira. Contudo, tais experiências, embora importantes e inovadoras, como é o caso dos sites governamentais, possuem pouco espectro de ação, diante da fragilidade histórica 
dos produtores familiares, em especial do semi-árido, no que se refere à infraestrutura, crédito, logística, acesso à mercados e organização social.

Por fim, não há como relacionar SAN e agricultura familiar, sem trazer luz para o papel estratégico das mulheres camponesas ${ }^{8}$ e seus múltiplos olhares para a segurança familiar e nutricional. Fortemente vinculada à obtenção de alimentos, as mulheres rurais têm papel central na manutenção dos quintais agroecológicos, onde se encontra grande parte da "comida de verdade" da família, tendo também um impacto econômico na família, pois é o alimento que "se deixa de comprar". Estão envolvidas na criação de pequenos animais e em situações de escassez hídrica, são as mulheres que vão em busca de água. Seu papel se estende na questão da soberania, por meio da manutenção de sementes e mudas, protagonizando processos de troca e atuando como guardiãs de muitas espécies (SILIPRANDI, 2015).

Segundo o Fórum Brasileiro de Soberania e Segurança Alimentar e Nutricional (FBSSAN, 2020a), as respostas dos governos sobre medidas de enfrentamento da fome têm sido lentas e confusas. É mencionada a implementação desigual da entrega de kits escolares com recursos do Programa Nacional de Alimentação Escolar e a falta de ações em prol do Programa de Aquisição de Alimentos, tornando agricultores inseguros quanto à venda de suas produções. Baseados nesta questão, o FBBSSAN, em parceria com a Campanha Nacional pelo Direito à Educação e o Movimento Sem Terra (MST), elaborou o Guia “Alimentação Escolar no contexto da pandemia da COVID-19". O guia aborda a garantia de compra de $30 \%$ da agricultura familiar, a qualidade nutricional dos alimentos distribuídos, apresenta boas experiências de implementação dos programas e traz orientações para agentes de SAN no monitoramento da alimentação escolar durante a pandemia. No site do Fórum também está disponível um material contendo orientações para a criação de Comitês Emergenciais de Combate à fome por Soberania e Segurança Alimentar e Nutricional nos estados e municípios, elaborado pela comissão organizadora da Conferência Popular por Soberania e Segurança Alimentar e Nutricional para apoiar a articulação e o fortalecimento das ações locais de SAN durante a pandemia (FBSSAN, 2020a,b).

Ações como as citadas são fundamentais para capilarizar as ações e chegar aos grupos populacionais vulnerabilizados. Mesmo as políticas públicas com estratégias direcionadas, que são essenciais e necessárias para minimizar os impactos em tempos de crise, produzem pontos

\footnotetext{
${ }^{8}$ Essa reflexão pode ser estendida para as mulheres do mar (pescadoras, marisqueiras), povos das florestas e dos demais ecossistemas.
} 
cegos e lacunas de cobertura sobre o território e suas populações (PIRES, 2020). A exemplo disso está o auxílio emergencial financeiro concedido pelo governo federal brasileiro, destinado aos trabalhadores informais, microempreendedores individuais, autônomos e desempregados. Entre as regras para recebê-lo é preciso que o Cadastro de Pessoas Física (CPF) esteja regularizado e a realização do cadastro para recebê-lo é feita em plataforma digital. Pessoas com irregularidades familiares e sem acesso à tecnologia não conseguem aprovação. $\mathrm{O}$ que dizer das pessoas sem endereço, que vivem nas ruas? Diante desse contexto, estratégias de organização social necessitam ser revistas e até permanentemente modificadas para que as pessoas se alimentem de forma digna.

\section{COVID-19 em Portugal: novos desafios ao abastecimento e consumo alimentares}

Desafios em termos de logística, envolvendo o movimento de alimentos, têm sido resolvidos, mas diversos constrangimentos sentem-se em alguns setores, particularmente desde o início de maio, como a produção pecuária devido à redução do acesso à alimentação animal e à capacidade reduzida dos matadouros (devido à restrições logísticas e escassez de mão-deobra). O bloqueio nas rotas logísticas de transporte é particularmente evidente nas cadeias de suprimento de alimentos frescos e resultam também em níveis superiores de perda e desperdício de alimento, em particular no que diz respeito aos produtos perecíveis. As restrições de transporte e as medidas de quarentena têm vindo a impedir o acesso de agricultores e pescadores aos mercados, restringindo suas capacidades produtivas e impedindo-os de vender seus produtos (FAO, 2020c)

A escassez de mão-de-obra é outro dos fatores que, no contexto da pandemia, tem vindo a atrapalhar a produção e o processamento de alimentos, principalmente para indústrias de trabalho intensivo (por exemplo, culturas de alto valor, carne e peixe), em particular nos períodos de isolamento social obrigatórios por determinação política.

De igual modo, o encerramento de restaurantes, lojas de comida de rua e mercados locais reduz um mercado importante para muitos agricultores familiares originando um excesso temporário de produção. De fato, devido à pandemia provocada pelo novo coronavírus, de modo a conter a sua transmissão, várias medidas foram decretadas, restringindo a mobilidade e a iniciativa econômica, na expectativa de se evitar, a todo o custo, deslocações fora do domicílio. Nesta continuidade, novos comportamentos alimentares surgiram na tentativa do 
consumidor proceder ao melhor ajuste, à melhor adaptação no que diz respeito ao que comer e onde comprar os alimentos.

Em Portugal, estas alterações decorreram essencialmente de uma nova realidade: o recolhimento domiciliário obrigatório e o tele trabalho. Numa primeira fase, mesmo antes do anúncio do estado de emergência, este consumo realizado essencialmente em casa levou os consumidores a uma corrida aos locais de compra, nomeadamente aos supermercados, com vista a garantir o abastecimento de produtos essenciais, como alimentos menos perecíveis, logo passíveis de serem mais facilmente armazenados (caso das conservas, do arroz ou das massas) e produtos de higiene e limpeza (incluindo o "famoso" papel higiênico). Trata-se de um comportamento semelhante ao que ocorreu noutros países (ANDERSEN et al., 2020; BAKER et al., 2020; WATANABE, 2020) e que no fundo procurou abastecer de alimentos os agregados familiares, por um longo período. Naturalmente que os agregados que apresentaram menores custos de armazenagem, por possuírem dispensa ou arrecadação bem dimensionada e com boas condições de armazenamento, puderam reduzir as deslocações às lojas, comprando maior quantidade de produtos (BLATTBERG et al., 1978).

Progressivamente, assistiu-se, neste tempo de pandemia, a um crescimento da procura de alimentos pelos canais alternativos, nomeadamente o e-commerce com a entrega ao domicílio ou diretamente nas próprias lojas. Tradicionalmente, em Portugal, os produtos mais comprados online sejam artigos eletrônicos, seguidos de vestuário, viagens, livros, artigos de beleza (DELLOITE CONSULTORES, 2019). Neste tempo de pandemia, assistiu-se a um crescimento brusco da procura de alimentos pelos canais de e-commerce com a entrega ao domicílio ou diretamente nas próprias lojas, tendo-se registrado um crescimento nas compras de $52 \%$, no setor food delivery e take-away de refeições, e um crescimento de $45 \%$ nas compras online no setor do retalho/varejo alimentar (SIBS ANALYTICS, 2020), não obstante as dificuldades sentidas pelos maiores retalhistas/varejistas no início da pandemia, seja ao nível do funcionamento dos sítios Web, por exemplo, páginas que demoravam a carregar, ou dificuldades no agendamento de entregas (CONSULAI, 2020).

Neste contexto, cozinhar em casa permitiu às pessoas controlarem as suas ações porque manipulam e preparam os alimentos, ajudando a mitigar a percepção do risco, consubstanciado em sentimentos associados à falta de controle, ao elevado risco para as gerações futuras, à ocorrência de potenciais catástrofes, à consequências fatais, à inquietação ou mesmo ao medo (SLOVIC, 1987). Uma vez que o vírus se transmite por contato próximo de pessoa para pessoa, 
pelas gotículas emitidas pelos infectados quando tossem ou espirram e depois inaladas por outras pessoas, ou através de contato com objetos contaminados essencialmente através das mãos, recomenda-se que devem ser mantidas e reforçadas as medidas de prevenção de higiene pessoal e da cozinha (higienização das superfícies e das frutas, verduras e legumes consumidos crus), podendo haver a dúvida, a inquietação de que estas medidas não sejam tomadas em locais que os consumidores não controlam como é o caso dos restaurantes e dos bares (MILES; FREWER, 2001).

De fato, de acordo com estudo desenvolvido pela Direção Geral de Saúde (DGS) sobre alimentação e atividade física em contexto de contenção social, para os portugueses, as suas práticas de higiene dos alimentos foram alteradas com o novo coronavíus. Esta tomada de consciência sobre a necessidade de seguir certas recomendações, conduziu os portugueses a considerarem procurar mais informação sobre saúde e cuidados de saúde durante o período de isolamento no contexto da COVID-19, nomeadamente na área da alimentação, muito embora as pessoas com mais de 55 anos e com menor nível de escolaridade sentissem mais dificuldade em obter informações (PORTUGAL, 2020). Por outro lado, de acordo com as percepções dos consumidores portugueses, constata-se que os portugueses praticaram menos atividade física e aumentaram o consumo de snacks, doces, frutas e hortaliças durante o período de confinamento no âmbito da pandemia de COVID-19, embora fossem identificados diferentes padrões de alteração dos hábitos alimentares: por um lado, os consumidores que com a pandemia praticaram uma alimentação saudável, devido ao aumento do consumo de fruta, hortaliças e pescado, e por outro, os consumidores optaram por praticar uma alimentação não saudável, caracterizada pelo aumento de refeições pré-preparadas, snacks salgados, refrigerantes e takeaway e a uma diminuição de frutas e hortaliças. São essencialmente os homens, os mais jovens (16-34 anos), os inquiridos que não conheciam as orientações da DGS na área da alimentação, as pessoas com dificuldades econômicas e em situação de insegurança alimentar que mais adotaram o padrão de comportamento alimentar não saudável (PORTUGAL, 2020).

$\mathrm{Na}$ realidade, os fatores econômicos, essencialmente preço e rendimento/renda (RITSON; PETROVICI, 2001), assumem um papel importante na escolha dos produtos alimentares, particularmente para os consumidores de baixo rendimento/renda, dado serem mais sensíveis ao preço, na medida em que a escolha dos alimentos por parte destes consumidores está mais sujeita às flutuações dos preços, optando por soluções alimentares que 
melhor se ajustam aos seus orçamentos e que proporcionam maior saciedade (ARES et al., 2017; BURNS; COOK; MAVOA, 2013).

De fato, as famílias que auferem baixos rendimentos tendem a comprar menos frutas e vegetais e a comprar alimentos de elevada densidade energética. Duas principais razões explicam esta tendência: os alimentos de elevada densidade energética são mais palatáveis e são alimentos que fornecem grande quantidade de energia a um baixo custo (DREWNOWSKI, 2004; DREWNOWSKI; SPECTER, 2004).

\section{Considerações finais}

O objetivo do presente ensaio foi discutir a complexidade que implica um desastre ou emergência sanitária por um longo período. Tal situação faz com que se pense nas necessidades urgentes e a intervenção humanitária por parte dos governos ao longo prazo.

Como é bem conhecido, em qualquer emergência sanitária como a que está acontecendo atualmente, a falta de alimentos (fome) é a primeira consequência. Assim, a ajuda alimentar é necessária, mas não suficiente, pois é temporal, deve fazer parte de um conjunto de ações emergenciais, contudo uma ajuda alimentar somente não vai resolver problemas de insegurança alimentar, pois torna-se necessário a estruturação de estratégias a longo prazo.

Ainda que sejam observados esforços dos governos para atenuar os efeitos da pandemia na SAN das populações, é necessário compreender que seus efeitos são mais devastadores, porque as populações já acumulam um histórico de fragilidade no que se refere a SAN. Situações como a degradação ambiental, mudanças climáticas e falta de políticas públicas como alicerce de proteção social, além de constante violação do DHANA, tornam-se mais evidentes no contexto atual e são as bases para se repensar as ações governamentais para além de ações emergenciais, com resultados finitos e pouco eficientes para a dimensão do problema.

É importante que os esforços governamentais pensem as profundas brechas acumuladas no que se refere a alimentos e nutrição, essa vulnerabilidade emerge à medida que o confinamento se expande, com a deterioração da nutrição e das condições de saúde, em especial dos mais vulneráveis. Cabe lembrar que o pico dos problemas não será visto durante a emergência, mas poderá ser agravado no período pós-pandemia.

\section{Referências}

AFRICAN NEWS AGENCY. Rwanda to deliever free food to 20000 households during coronavirus lockdown. Africa Breefing, Cape Town, 30 mar. 2020. Disponível em: 
https://iol.co.za/news/africa/rwanda-to-deliever-free-food-20-000-households-duringcoronavirus-lockdown-45756889. Acesso em: 7 maio 2020.

ALENCASTRO, M. Pandemia atinge África de maneira desigual e reflete contraste entre países. Experiencias de Agola, Africa do Sul e Moçambique reveleam diferenças ao lidar com coronavirus. Folha de São Paulo, São Paulo, 12 abr. 2020. Disponível em:

https://www1 folha.uol.com.br/mundo/2020/04/pandemia-atinge-africa-de-maneira-desiguale-reflete-contraste-entre -paises.shtml. Acesso em: 13 jun. 2020.

ANDERSEN, A. L. et al. Consumer responses to the COVID-19 crisis: evidence from bank account transaction data. CEPR Discussion Papers, St Louis, n. DP14809, 2020. Disponível em: https://ssrn.com/abstract=3612880. Acesso em: 12 jun. 2020.

ARES, G. et al. Comparison of motives underlying food choice and barriers to healthy eating among low medium income consumers in Uruguay. Cad. Saúde Pública, Rio de Janeiro, v. 33, n. 4, p. e00213315, 2017.

ASSOCIAÇÃO BRASILEIRA DE NUTRIÇÃO. Garantir o direito à alimentação e combater a fome em tempos de coronavírus: a vida e a dignidade humana em primeiro lugar! Fóruns, redes, articulações, movimentos e organizações da sociedade civil. São Paulo: ASBRAN, 2020. Disponível em: https://www.cfn.org.br/wpcontent/uploads/2020/03/Recomenda\%C3\%A7\%C3\%B5es-SAN-e-Combate-a-fome-emtempos-de-Coronav\%C3\%ADrus-correto.pdf-2.pdf. Acesso em: 18 abr. 2020.

BAIARDI, A.; ALENCAR, C. M. M. Agricultura familiar, seu interesse acadêmico, sua lógica constitutiva e sua resiliência no Brasil. Rev. Econ. Sociol. Rural, Brasília, v. 52, p. 4562, 2014. Suplemento1.

BAKER, S. R. et al. How does household spending respond to an epidemic? Consumption during the 2020 COVID-19 pandemic. Rev. Asset Pricing Stud., Oxford, n. w26949, 2020.

BLATTBERG, R. et al. Identifying the deal prone segment. J. Mark. Res., Thousand Oaks, V. 15, n. 3, p. 369-377, 1978.

BURITY, V. Isolamento social ou combate à fome: o falso dilema em nossa mesa. Brasília: FIAN Brasil, 2020. Disponível em: https://fianbrasil.org.br/prato-do-diaisolamento-social-oucombate-a-fome-o-falso-dilema-em-nossa-mesa/. Acesso em: 20 abr. 2020.

BURITY, V.; FRANCESCHINI, T; VALENTE, F; RECINE, E.; LEÃO, M.; CARVALHO, $M$. de $F$. Direito humano à alimentação adequada no contexto da segurança alimentar $e$ nutricional. Brasília, DF: ABRANDH, p.1-204, 2010.

BURNS, C.; COOK, K.; MAVOA, H. Role of expendable income and price in food choice by low income families. Appetite, London, v. 71, p. 209-217, 2013.

CHAVEZ, N.; TELLEEN, S.; KIM, Y. O. R. Food insufficiency in urban latino families. J. Immigr. Minor. Health, New York, v. 9, p. 197-204, 2007.

CONSULAI. Resumo: agricultura em tempo de crise. Lisboa: Consulai, 2020. Disponível em: https://mcusercontent.com/c9e960e2338450a6e05d4c6ff/files/55a71cbb-faee-48de-bbb2f0a551794e5d/RESUMO_Debate_vf_compressed.pdf. Acesso em: 14 jun. 2020.

DANE. Departamento Administrativo Nacional de Estatística. Bogotá DC-Colómbia. Informação para todos. Large Integrated Household Survey (GEIH) Labor Market. 2020. 
Disponível em: https://www.dane.gov.co/index.php/estadisticas-por-tema/mercadolaboral/empleo-y-desempleo. Acesso em: 12 maio 2020.

DELOITTE CONSULTORES S.A. Obstáculos legais e concorrenciais ao desenvolvimento do e-commerce em Portugal. Londres, 2019. Disponível em: https://www2.deloitte.com/content/dam/Deloitte/pt/Documents/consumerbusiness/Deloitte_Obstaculos $\% 20 \mathrm{ao} \% 20$ desenvolvimento $\% 20 \mathrm{do} \% 20$ ecommerce.pdf. Acesso em: 13 jun. 2020.

DOMINGUES, F. S. A crise do corona vírus, segurança alimentar e abastecimento. Porto Alegre: UFRGS, 2020. Disponível em: https://www.ufrgs.br/fce/a-crise-do-corona-virusseguranca-alimentar-e-abastecimento/. Acesso em: 20 abr. 2020.

DREWNOWSKI, A. Obesity and the food environment: dietary energy density and diet costs. Am. J. Prev. Med., Amsterdam, v. 2, n. 3, p. 154-162, 2004

DREWNOWSKI, A.; SPECTER, S. E. Poverty and obesity: the role of energy density and energy costs. Am. J. Clin. Nutr., Rockville, v. 79, n. 1, p. 6-16, 2004.

FAO. Organización de las Naciones Unidas para la Alimentación y la Agricultura. La seguridad alimentaria y la nutrición en el mundo: protegerse frente a la desaceleración y el debilitamiento de la economía. Roma, 2019. Disponível em:

http://www.fao.org/3/ca5162es/ca5162es.pdf. Acesso em: 28 abr. 2020.

FAO. Organização das Nações Unidas para Alimentação e Agricultura. Migración. Roma, 2020a. Disponível em: http://www.fao.org/migration/es/. Acesso em: 13 jun. 2020.

FAO. Organización de las Naciones Unidas para la Alimentación y la Agricultura. Sistemas agroalimentares e de seguridad alimentaria e nutricional. Reporte FAO COVID-19 \#3. Roma: FAO, 2020b.

FAO. Organização das Nações Unidas para Alimentação e Agricultura. food price monitoring and analysis. Rome: FAO, 2020c. Disponível em:

http://www.fao.org/giews/food-prices/home/en/. Acesso em: 15 jun.2020.

FAO. African Union. Reunião de Ministros da Agricultura africanos. Declaração sobre segurança alimentar e nutrição, durante a pandemia da COVID-19. Roma: FAO, 2020d. p. 1-7.

FAO. Organização das Nações Unidas para Alimentação e Agricultura. América Latina e Caribe. FAO faz alerta sobre o impacto do COVID19 na alimentação escolar na América Latina e no Caribe. Roma: FAO, 2020e. Disponível em: http://www.fao.org/americas/noticias/ver/pt/c/1267030/. Acesso em: 20 abr. 2020.

FAO. Brasil. Organização das Nações Unidas para Alimentação e Agricultura. Brasil. Atenuar os efeitos do Covid-19 no comércio e nos mercados de alimentos. Roma: FAO, 2020f. Disponível em: http://www.fao.org/brasil/noticias/detail-events/pt/c/1269086/. Acesso em: 20 abr. 2020.

FAO. Organización de las Naciones Unidas para la Alimentación y la Agricultura; CELAC (Comunidad de Estados Latinoamericanos y Caribeños). Nuevo informe de la FAO advierte del impacto del COVID-19 en la seguridad alimentaria de América Latina y el Caribe. 
Santiago: CELAC, 2020g. Disponível em:

http://www.fao.org/americas/noticias/ver/es/c/1272991/. Acesso em: 14 jun. 2020.

FIOCRUZ. Fundação Oswaldo Cruz. Sala de Convidados debate coronavírus e segurança alimentar. Rio de Janeiro: FIOCRUZ, 2020. Disponível em:

https://portal.fiocruz.br/noticia/sala-de-convidados-debate-coronavirus-e-seguranca-alimentar. Acesso em: 20 abr. 2020.

FORMAN, S. Camponeses: sua participação no Brasil. Tradução: Maria Isabel Erthal Abdenur. Rio de Janeiro: Paz e Terra, 1979. Título original: The Brazilian Peasantry.

FÓRUM BRASILEIRO DE SOBERANIA E SEGURANÇA ALIMENTAR E NUTRICIONAL. Guia COVID-19: alimentação escolar. Rio de Janeiro: FBSSAN, 2020a. Disponível em: https://fbssan.org.br/2020/05/guia-para-alimentacao-escolar-em-tempos-decovid-19/. Acesso em: 22 abr. 2020.

FÓRUM BRASILEIRO DE SOBERANIA E SEGURANÇA ALIMENTAR E NUTRICIONAL. Como nos organizar para exigir o direito à alimentação e combater a Fome em tempos de coronavírus. Rio de Janeiro: FBSSAN, 2020b. Disponível em: https://fbssan.org.br/2020/04/orientacoes-para-criacao-de-comites-emergenciais/. Acesso em: 22 abr. 2020.

GUIMARÃES, R. M. Os impactos das políticas de austeridade nas condições de saúde dos países com algum tipo de crise. Trab. Educ. Saude, Rio de Janeiro, v. 16, n. 1, p. 383-385, 2018.

INSTITUTO BRASILEIRO DE DEFESA DO CONSUMIDOR. Plataforma ajuda a encontrar comida de verdade durante pandemia. São Paulo: IDEC, 2020. Disponível em: https://idec.org.br/noticia/idec-divulga-iniciativas-que-vendem-alimentos-saudaveis-durantepandemia. Acesso em: 20 abr. 2020

LUCENA, C. C.; HOLANDA, F. Z. F.; BOMFIM, M. A. D. Atuais e potenciais impactos do coronavírus (Covid-19) na caprinocultura e ovinocultura. Sobral: Centro de Inteligência e Mercado de Caprinos e Ovinos, Sobral, 2020. (Boletim n. 10).

MACEDO, Y. M.; ORNELLAS, J. L.; BOMFIM, H. F. COVID-19 nas favelas e periferias brasileiras. BOCA Bol. Conjunt., Boa Vista, v. 2, n. 4, p. 50-54, 2020.

MASIPA, T. S. The impact of climate change on food security in South África: current realizes and Challenges ahead. J. Disastre Risk Stud., Jàmbá, v. 9, n. 1, p. 411, 2017. DOI 10.4102/jamba.v9i1.411. eCollection 2017.

MELLO-THÉRY, N.; THÉRY, H. A geopolítica do COVID-19. Rev. Bras. Geogr. Econ., Rio de Janeiro, v. 9, n. 17, p. 1-9, 2020.

MENHAS, R.; UMER, S.; SHABBIR, G. Climate change and its impact food and nutrition security in Pakistan. Iran J. Public Health, Tehran, v. 45, n. 4, p. 551-2016.

MILES, S.; FREWER L. J. Investigating specific concerns about different food hazards. Food Quality and Preference, Harlow, v. 12, p. 47-61, 2001.

OLIVEIRA, T. C.; ABRANCHES, M. V.; LANA, R. M. (In)Segurança alimentar no contexto da pandemia por SARS-CoV-2. Cad. Saúde Pública, Rio de Janeiro, v. 36, n. 4, p. e00055220, 2020. 
ONU. Organização das Nações Unidas. Human rights dimensions of COVID-19 Response. New York: ONU, 2020. Disponível em: https:/www.hrv.org/news/2020/03/19/human-rightsdimensions-covid-19-response. Acesso em: 28 maio 2020.

ONU. Organização das Nações Unidas. Fome aumenta no mundo e atinge 820 milhões de pessoas, diz relatório da ONU. 15 jul 2019. Disponível em: https://nacoesunidas.org/fomeaumenta-no-mundo-e-atinge-820-milhoes-de-pessoas-diz-relatorio-da-onu/. Acesso em: 10 jun. 2020.

ONU. Organização das Nações Unidas. Pacto internacional dos direitos econômicos, sociais e culturais das Nações Unidas. New York: ONU, 1966. Disponível em: https://reaties.un.org/doc/Publication/MTDSG/Volume\%20I/chapter\%20IV/IV-3.en.pdf. Acesso em: 10 jun. 2020.

PIRES, R. R. C. Os efeitos sobre grupos sociais e territórios vulnerabilizados das medidas de enfrentamento à crise sanitária da COVID-19: propostas para o aperfeiçoamento da ação pública. Nota Técnica n. 33. Brasília: Instituto de Pesquisa Econômica Aplicada. Diretoria de Estudos e Políticas do Estado, das Instituições e da Democracia, 2020. Disponível em:

http://repositorio.ipea.gov.br/bitstream/11058/9839/1/NT_33_Diest_Os\%20Efeitos\%20Sobre $\% 20$ Grupos $\% 20$ Sociais $\% 20 \mathrm{e} \% 20$ Territ $\%$ c3\%b3rios $\% 20 \bar{V}$ ulnerabilizados.pdf. Acesso em: 20 abr. 2020.

PORTUGAL. Direção-Geral de Saúde. REACT-COVID: inquérito sobre alimentação e atividade física em contexto de contenção social. Lisboa, 2020. Disponível em:

https://www.dgs.pt/documentos-e-publicacoes/inquerito-sobre-alimentacao-e-atividade-fisicaem-contexto-de-contencao-social.aspx. Acesso em: 14 jun. 2020.

POTOCHNICK, S. et al. Food insecurity among hispanic/Latino youth: Who is at risk and what are the health correlates? J. Adolesc. Health, New York, v. 64, n. 5, p. 631-639, 2019.

RITSON, C.; PETROVICI, D. The economics of food choice: is price important? In: FREWER, L. J.; RISVIK, E.; SCHIFFERSTEIN, H. (ed.). Food, people and society. Berlin: Springer, 2001. p. 339-363.

SANTOS, I. S.; VIEIRA, F. S. Direito à saúde e austeridade fiscal: o caso brasileiro em perspectiva internacional. Ciênc. Saude Colet., Rio de Janeiro, v. 23, n. 7, p. 2303-2314, 2018.

SIBS ANALYTICS. O impacto do coronavírus no hábito de consumo dos portugueses. 2020. Disponível em: https://www.sibsanalytics.com/noticias/o-impacto-do-coronavirus-noshabitos-de-consumo-dos-portugueses/. Acesso em: 13 jun. 2020.

SILIPRANDI, E. Mulheres e agroecologia: transformando o campo, as florestas e as pessoas. Rio de Janeiro: Editora UFRJ, 2015.

SLOVIC, P. Perception of risk. Science, New York, v. 236, p. 280-285, 1987.

TONG, S. et al. Climate change, food, water and population health in China. Bull. World Health Organ., Geneva, v. 94, p. 759-765, 2016.

WATANABE, T. The responses of consumption and prices in Japan to the COVID-19 crisis and the Tohoku Earthquake. Tokyo: University of Tokyo, Graduate School of 
Economics, 2020. (Working Papers on Central Bank Communication, 020). Disponível em: https://academiccommons.columbia.edu/doi/10.7916/d8-qs4v-q792. Acesso em: 11 jun. 2020.

\title{
FOOD AND NUTRITIONAL SECURITY IN COVID-19 TIMES: IMPACTS IN AFRICA, LATIN AMERICA AND PORTUGAL
}

\begin{abstract}
Economic and sanitary crises have great repercussions on access to food and the health of the population. In this context, austerity policies can have devastating effects on social rights. The purpose of this essay is to reflect on the impact of various types of crises on the Human Right to Adequate Food and Nutrition (DHANA) and on Food and Nutritional Security (SAN) of vulnerable populations and to identify of public policies, programs and actions developed based on the relief of the damage caused by the COVID-19 pandemic. From the collection of information carried out in a non-systematic way on government websites and scientific databases, documents were read in full, categorized, and critically analyzed. Three different contexts, Africa, Latin America, and Portugal, are presented by the authors who work in these realities, with the intention of contributing to mitigate the food problems that worsen and are derived from the pandemic situation.
\end{abstract}

Keywords: Consumption; COVID-19; Right to Food; Food and nutrition security; Pandemic.

\section{SEGURIDAD ALIMENTARIA Y NUTRICIONAL EN TIEMPOS DE COVID 19: IMPACTOS EN ÁFRICA, AMÉRICA LATINA Y PORTUGAL}

\begin{abstract}
Resumen
Las crisis económicas y sanitarias tienen grandes repercusiones en el acceso a los alimentos y la salud de la población. En este contexto, las políticas de austeridad pueden tener efectos devastadores en los mecanismos para garantizar los derechos sociales. El objetivo de este ensayo es reflexionar sobre el impacto de diversos tipos de crisis en el Derecho Humano a la Alimentación y Nutrición Adecuada (DHANA) y en la Seguridad Alimentaria y Nutricional (SAN) de poblaciones vulnerables, así como la identificación de las políticas públicas, programas y acciones desarrolladas tomando como referencia el alivio de los daños causados por la pandemia del COVID-19. A partir de la recolección de información realizada de forma no sistemática fueron leídos los documentos de forma íntegra, categorizados y analizados críticamente a partir de textos en sitios no gubernamentales y bases de datos científicas. Los autores que trabajan en estas realidades presentan tres contextos distintos, África, América Latina y Portugal, con la intención de contribuir a mitigar los problemas alimentarios que empeoran y se derivan de la situación pandémica.
\end{abstract}

Palabras claves: Consumo; COVID-19; Derecho a la Alimentación; Seguridad Alimentaria y Nutricional; Pandemia. 